\title{
Pyeloduodenal Fistula \\ in Xanthogranulomatous Pyelonephritis
}

\author{
Ryan Yan, Mark T. Dawidek, Drew Phillips, Ben H. Chew $\bowtie$ \\ Department of Urologic Sciences, University of British Columbia, Vancouver, Canada
}

Xanthogranulomatous pyelonephritis (XGP) is a rare but severe form of inflammatory renal disease associated with renal stones and results in granulomatous tissue invading and destroying normal parenchyma. A serious complication of XGP is fistulization to surrounding structures. We present a case of XGP with pyeloduodenal fistula (PDF) in a 48-year-old female with a history of hypertension and recurrent urinary infections but no previous stones.

The patient initially presented to the emergency room in March 2020 with flank pain, nausea, hematuria, and weight loss. Abdominal CT revealed right-sided 4 $\mathrm{cm}$ partial staghorn and $2.7 \mathrm{~cm}$ ureteropelvic junction stones. There was no abscess and there were no signs of XGP. The patient was referred to our center in April and booked for percutaneous nephrolithotomy (PCNL). Her procedure was delayed because of the COVID-19 pandemic. On May 15, she developed Proteus mirabilis urosepsis and new renal abscesses. She was admitted to a peripheral hospital and underwent ureteroscopy and ureteric stent insertion for purulent drainage.

On May 27, she presented for PCNL, and the retrograde pyelogram showed communication of the kidney with the duodenum (Figure 1A). The procedure was aborted, and an urgent esophagoduodenoscopy confirmed the diagnosis of PDF. The patient underwent an open simple nephrectomy and Roux-en-Y duodenojejunostomy with loop gastrojejunostomy and pyloric exclusion. The PDF was identified with a traversing ureteric stent intraoperatively (Figure 1B). The remainder of her course in hospital was unremarkable and there have been no further urinary infections or stones after one year follow-up.
Final pathology revealed diffuse xanthogranulomatous inflammation of the kidney invading through the duodenum. XGP is challenging to diagnose and PDFs are exceedingly rare. Only a handful of cases have been reported[1-3]. This case was remarkable for the rapid progression from staghorn renal calculus to fistulizing XGP over a matter of months.

\section{FIGURE $1 \mathrm{~A}$.}

Retrograde pyelogram with ureteral catheter in right renal pelvis and contrast collecting in duodenum and stomach

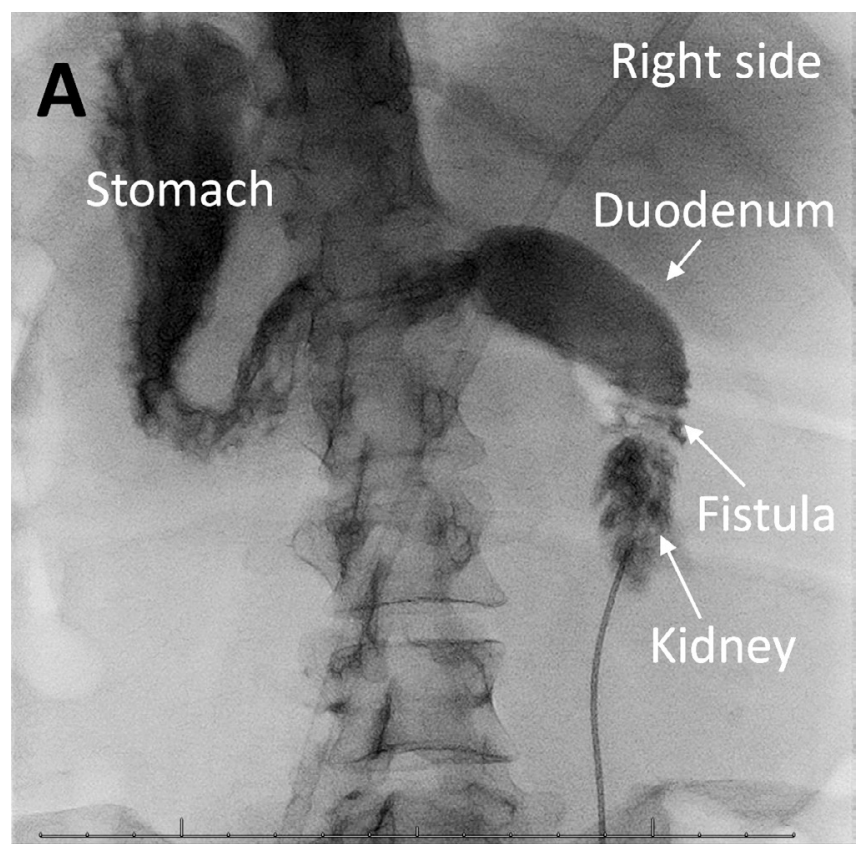

\section{Key Words}

Xanthogranulomatous pyelonephritis, staghorn calculus, pyeloduodenal fistula, proteus mirabilis, XGP

\section{Competing Interests}

None declared

Patient Consent: Obtained.

\section{Article Information}

Received on June 3, 2021

Accepted on June 5, 2021

Soc Int Urol J.2021;2(4):265-266

DOI: $10.48083 /$ NSLU7720 


\section{FIGURE 1B.}

Intraoperative photograph after transecting the fistula tract revealing a ureteral double-J stent traversing from the right renal pelvis to the duodenum

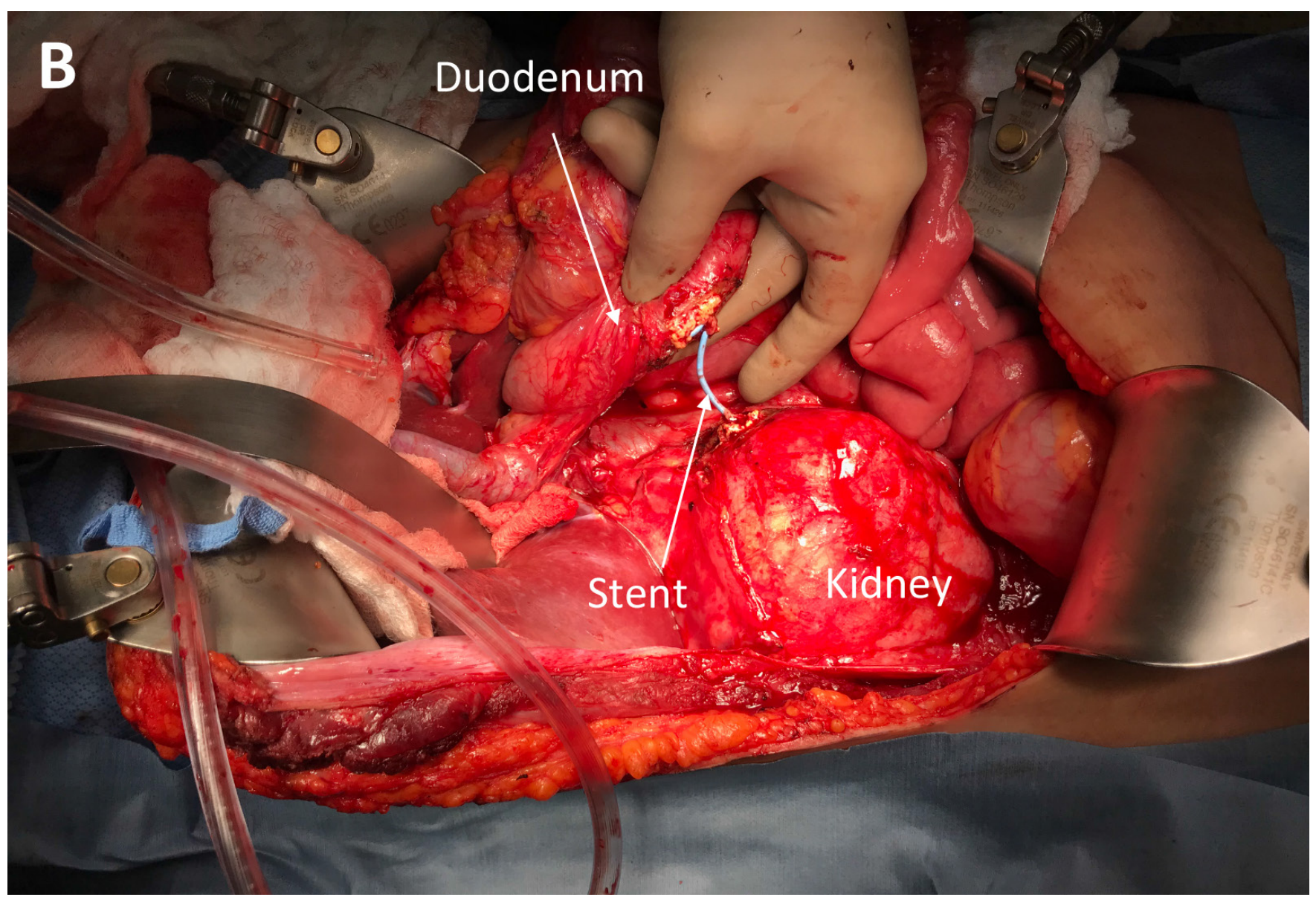

\section{References}

1. Cheatle TR, Waldron RP, Arkell DG. Xanthogranulomatous pyelonephritis associated with pyeloduodenal fistula. Br J Surg.1985 Sep;72(9):764. doi: 10.1002/bjs.1800720934.

2. Sallami S, Rhouma S Ben, Kchir N, Dagudagui N, Nouira Y, Horchani A. Spontaneous pyeloduodenal fistula complicating a xanthogranulomatous pyelonephritis: a case report. UroToday Int J.2010 Apr;3(2). doi:10.3834/uij.1944-5784.2010.04.09
3. Laberge M, Kulkarni GS, Sreeharsha B. Pyeloduodenal fistula complicating xanthogranulomatous pyelonephritis. Int Urol Nephrol.2018;50(6):1071-3. doi.org/10.1007/s11255-018-1886-x 